\title{
Surgical treatment for medically refractory myasthenic blepharoptosis
}

\author{
This article was published in the following Dove Press journal: \\ Clinical Ophthalmology \\ 19 September 2014 \\ Number of times this article has been viewed
}

\author{
Yusuke Shimizu' \\ Shigeaki Suzuki \\ Tomohisa Nagasao' \\ Hisao Ogata' \\ Masaki Yazawa' \\ Norihiro Suzuki ${ }^{2}$ \\ Kazuo Kishi' \\ 'Department of Plastic and \\ Reconstructive Surgery, ${ }^{2}$ Department \\ of Neurology, Keio University School \\ of Medicine, Tokyo, Japan
}

Purpose: Currently, only a few reports have recommended surgery as a suitable treatment for blepharoptosis associated with myasthenia gravis. The present study aims to introduce our surgical criteria, surgical options, outcomes, and precautions for medically refractory myasthenic blepharoptosis.

Patients and methods: Eight patients who failed to respond to at least 2 years of medical treatment and who underwent blepharoptosis surgery, from January 2008 to December 2011, were enrolled in this study. Medical records, photographs, and questionnaire results regarding postoperative status were evaluated. Of the eleven procedures performed, four involved frontal suspension, four involved external levator advancement, one involved nonincisional transconjunctival levator advancement, and two involved subbrow blepharoplasty with orbicularis oculi muscle tucking. The margin reflex distance improved postoperatively in seven patients.

Results: Seven patients had very minimal scarring, and one had minimal scarring. Five patients showed no eyelid asymmetry, one had subtle asymmetry, and two had obvious asymmetry. Seven patients were very satisfied, and one patient was satisfied with the overall result. Postoperative complications included mild lid lag with incomplete eyelid closure, prolonged scar redness, and worsened heterophoria. No patient experienced postoperative exposure keratitis or recurrent blepharoptosis during the study period.

Conclusion: Our results indicate that blepharoptosis surgery is effective for patients with myasthenia gravis, especially those with residual blepharoptosis despite multiple sessions of medical treatments. We recommend that neurologists and surgeons collaborate more systematically and discuss comprehensive treatment plans to increase the quality of life for patients with myasthenia gravis.

Keywords: ptosis, myasthenia gravis, ocular myasthenia, blepharoplasty

\section{Introduction}

At present, many studies of blepharoptosis surgery have been published. However, few reports have described specific procedures or precautions for blepharoptosis surgery in patients with myasthenia gravis $(\mathrm{MG}) .{ }^{1-5} \mathrm{MG}$ is an autoimmune disorder in which the body's own antibodies block the transmission of nerve impulses to muscles, causing fluctuating weakness and muscle fatigue. ${ }^{6}$ Based on retrospective case reviews, blepharoptosis is the first sign of MG in approximately $50 \%$ of patients, ${ }^{7-9}$ and more than $90 \%$ of patients experience ocular symptoms during the course of their disease. ${ }^{10}$ To improve the quality of vision, medical treatment is administered first, typically with acetylcholine esterase inhibitors (AchE-I). However, some patients require additional therapy because of the limited efficacy of AcheE-I in some patients. Moreover, while corticosteroids are very effective in the short term, long-term adverse effects occur in up to $38 \%$ of cases. ${ }^{11,12}$ Thus, neurologists often struggle to treat patients with medically refractory longstanding blepharoptosis. ${ }^{13-15}$
Correspondence: Yusuke Shimizu Department of Plastic and Reconstructive Surgery, Keio University School of Medicine, 35 Shinanomachi, Shinjuku,

Tokyo 160-8582, Japan

Tel +8I 353633814

Fax +8I 333521054

Email yyssprs@gmail.com 
In such cases, an oculoplastic surgeon may collaborate with neurologists to perform blepharoptosis surgery. However, a recent Japanese multicenter survey noted a very low rate of blepharoptosis surgery in patients with $\mathrm{MG}$ (19 out of $676[2.8 \%]) .{ }^{16}$ Through surveys and interviews ${ }^{11}$ at Japanese neurological centers, we found that neurologists often do not closely collaborate with plastic or oculoplastic surgeons in cases of myasthenic blepharoptosis. In general, neurologists are understandably unfamiliar with the efficacy and drawbacks of blepharoptosis surgery and may hesitate to recommend this as a surgical option.

The present report aims to acquaint neurologists with the efficacy and drawbacks of blepharoptosis surgery, in an attempt to encourage a closer relationship with oculoplastic surgeons. In addition, to inform surgeons about the surgical approaches for medically refractory longstanding myasthenic blepharoptosis, this report presents our criteria, procedure options, outcomes, and precautions of the surgery, from our experiences.

\section{Materials and methods Patients}

We examined 220 Japanese patients who were definitively diagnosed with MG at the MG clinic of Keio University Hospital, from January 2008 to December 2011. Among them, eight patients $(3.6 \%)$ who failed to respond to medical treatment and underwent blepharoptosis surgery were enrolled in the study. Three patients who underwent blepharoptosis surgery but whose MG was not followed at the clinic were excluded from the study. The eight patients were also among 19 surgical patients described in a previous Japanese multicenter study. ${ }^{16}$ All patients were followed for at least 2 years. All clinical information was collected after the patients had provided written informed consent, and the study was approved by the institutional review board of Keio University, School of Medicine.

\section{Methods}

Medical records and photographs pertaining to patients' blepharoptosis surgery were reviewed. The pre- and 1-year postoperative margin reflex distance (MRD) at the time of most severe MG were measured. Questionnaires were also given to the patients concerning the ease of eyelid opening (over both the short term [ $\leq 1$ year] and long term [ $>1$ year] postoperatively), esthetic outcome (scarring, asymmetry), overall satisfaction with the surgery, and any noted complications. Three-point Likert scales were used to assess the ease of eyelid opening (much easier, easier, worse), scarring (very minimal, minimal, visible), eyelid asymmetry (none, subtle, obvious), and overall satisfaction with the surgery (very satisfied, satisfied, dissatisfied). To evaluate scarring, the patients answered the questionnaires with their attending neurologists at the outpatient clinics. To encourage unbiased opinions from the patients, the surgeons were not present at questionnaire completion.

\section{Surgical criteria}

If a patient with blepharoptosis with an etiology suggestive of MG was first referred to the plastic surgery department rather than the neurology department, then the attending plastic surgeon consistently consulted a neurologist. Therefore, none of the patients underwent blepharoptosis surgery before receiving a definitive diagnosis of MG. Patient selection for surgical treatment was carefully judged by the attending neurologist according to the following four criteria: (1) stable general condition, (2) at least 2 years of prior medical treatment by neurologists before surgery, (3) continuous blepharoptosis with minimal circadian changes, and (4) quality of life severely disrupted by blepharoptosis (Table 1).

\section{Surgical options}

The surgical procedure was selected through discussion among several board-certified plastic surgeons, in light of the patient's condition. The surgical options used at Keio University Hospital are illustrated in Figure 1. These surgical procedure options are basically the same as those used for blepharoptosis surgery without MG. Although the final decision was always made after careful consultation with the patient, we placed a significant emphasis on levator function when MG was most severe, as the primary factor for determining the choice of surgical procedure. In addition, MRD, skin thickness and redundancy, and patient preference were taken into account. Eyebrow height, lash-brow distance, bilateral difference in orbital shape, coexisting disease other than MG, and medical therapies, such as antiplatelet agents, were also considered.

Table I Surgical criteria for myasthenic blepharoptosis of Keio University Hospital

\begin{tabular}{ll}
\hline Element & Content \\
\hline General condition & Stable \\
Medical treatment & $\begin{array}{l}\text { At least 2 years of prior medical treatment } \\
\text { before surgery } \\
\text { Circadian change }\end{array}$ \\
Quality of life & $\begin{array}{l}\text { continuous blepharoptosis, with minimal } \\
\text { Severely disrupted }\end{array}$ \\
\hline
\end{tabular}




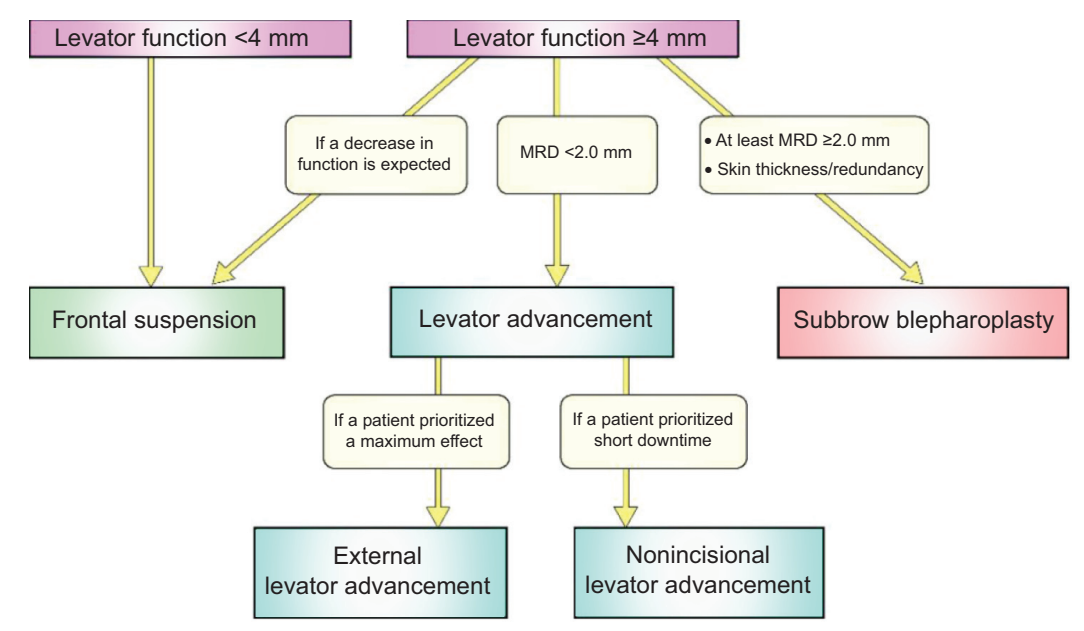

Figure I The surgical options used for blepharoptosis surgery at Keio University Hospital.

\section{Surgical procedure}

We used several surgical procedures to treat blepharoptosis in patients with MG: frontal suspension, external levator advancement, nonincisional levator advancement, and subbrow blepharoplasty. In frontal suspension, we preferably used autogenous fascia lata. The harvested fascia lata was tailored to an inverted $\mathrm{Y}$ shape, and the separated caudal legs were then fixed to the tarsus, while the cephalic end was grafted inside the eyebrow through a suborbital septum tunnel. In cases where the fascia lata was avoided, several nylon or GORE-TEX ${ }^{\circledR}$ sutures were used to suspend the eyelid from the eyebrow.

In external levator advancement, we used the classic transcutaneous levator advancement technique described by Carraway and Vincent, ${ }^{17}$ which has broad utility. In nonincisional levator advancement, we used a transconjunctival levator advancement technique, ${ }^{18}$ which has the potential to reduce postoperative downtime since no major incision of the skin nor conjunctiva is required. In subbrow blepharoplasty, we removed a certain amount of skin and orbicularis oculi muscle to reduce lateral hooding and the weight of the upper eyelid skin mounted on to the eyelash. The orbicularis oculi muscle was tucked to elevate the eyelid margin.

\section{Results}

Eleven blepharoptosis surgeries were performed on eleven eyes of eight patients. Three patients were male, and five were female. The age at operation ranged from 38 to 78 years (mean 67.4 years, median 76 years). The age at $\mathrm{MG}$ diagnosis ranged from 22 to 75 years (mean 56.6 years, median 63.5 years). The time to surgery after MG diagnosis ranged from 2 to 30 years (mean 10.1 years, median 5 years). The clinical features of the patients with MG are outlined in Table 2.
Of the eleven procedures performed, four involved frontal suspension, four involved external levator advancement, one involved nonincisional transconjunctival levator advancement, and two involved subbrow blepharoplasty with orbicularis oculi muscle tucking. MRD improved postoperatively in all patients except patient 7 , who underwent subbrow blepharoplasty. The surgical outcomes of the patients with MG are presented in Table 3.

Postoperative complications were noted in several patients, including mild lid lag with incomplete eyelid closure, prolonged scar redness, and worsened heterophoria. No patients presented postoperative corneal exposure keratitis, and none underwent surgery for overcorrection. Although distinct recurrent ptosis did not develop in any patients during the study period, one patient (patient 2) underwent additional skin resection surgery, to achieve the maximum effect of eyelid elevation, 25 months after the initial surgery.

\section{Case reports}

\section{Case 2}

A 71-year-old man was diagnosed with MG after developing right blepharoptosis and diplopia. The Myasthenia Gravis Foundation of America (MGFA) grade was 1 (quantitative myasthenia gravis [QMG] score of 7, at its most severe). ${ }^{19}$ Although AchE-I slightly improved the ptosis, severe blepharoptosis remained, especially in the evening. At 73 years of age, the patient also developed right Bell's palsy, which caused persistent right brow ptosis and paretic ocular synkinesis. This combination of blepharoptosis and brow ptosis severely disrupted the patient's quality of vision. At 75 years of age, the patient was referred to the plastic surgery department. Levator function was $12 \mathrm{~mm}$, and MRD was $0 \mathrm{~mm}$ (Figure 2A). Since the patient was not particularly 

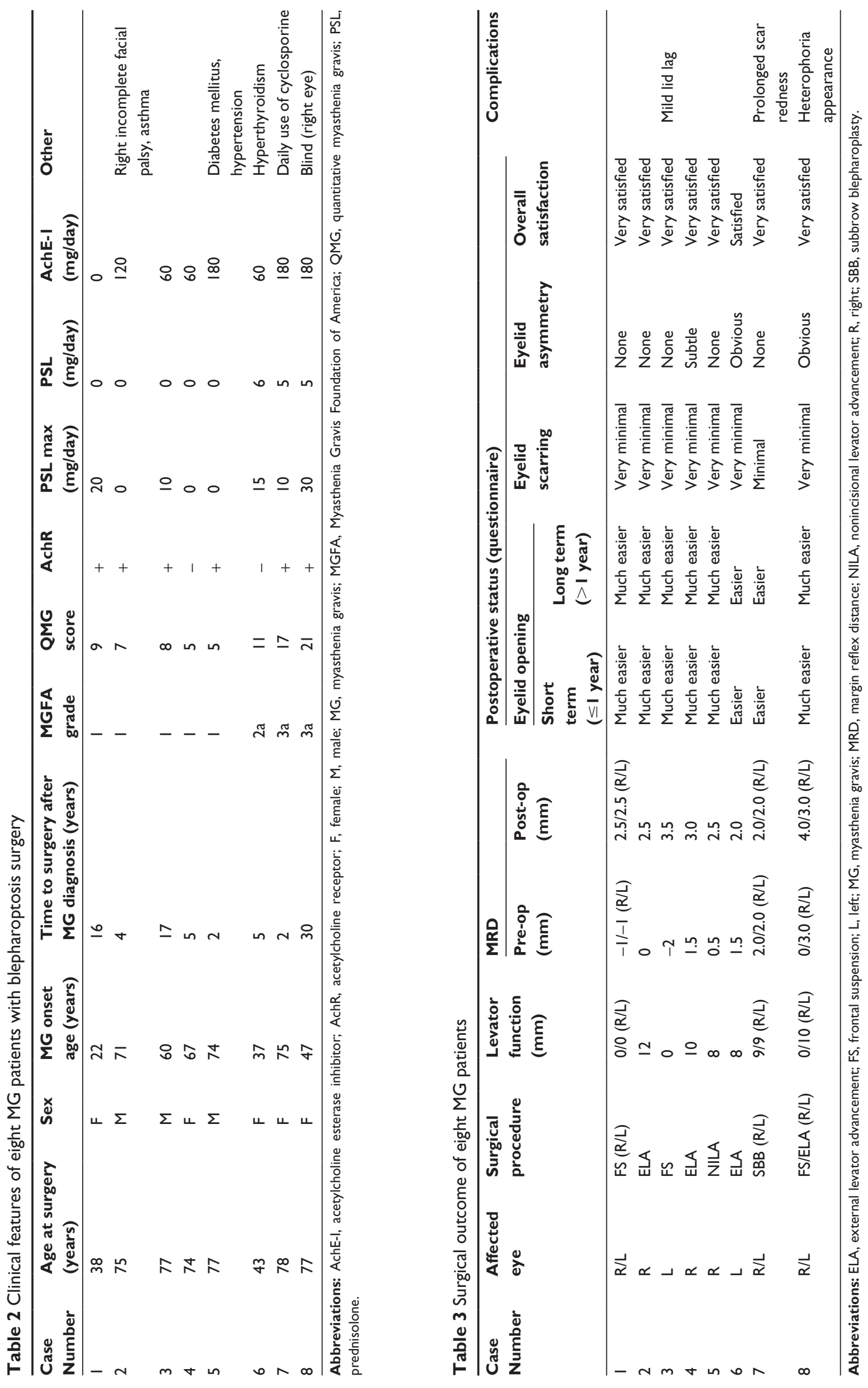

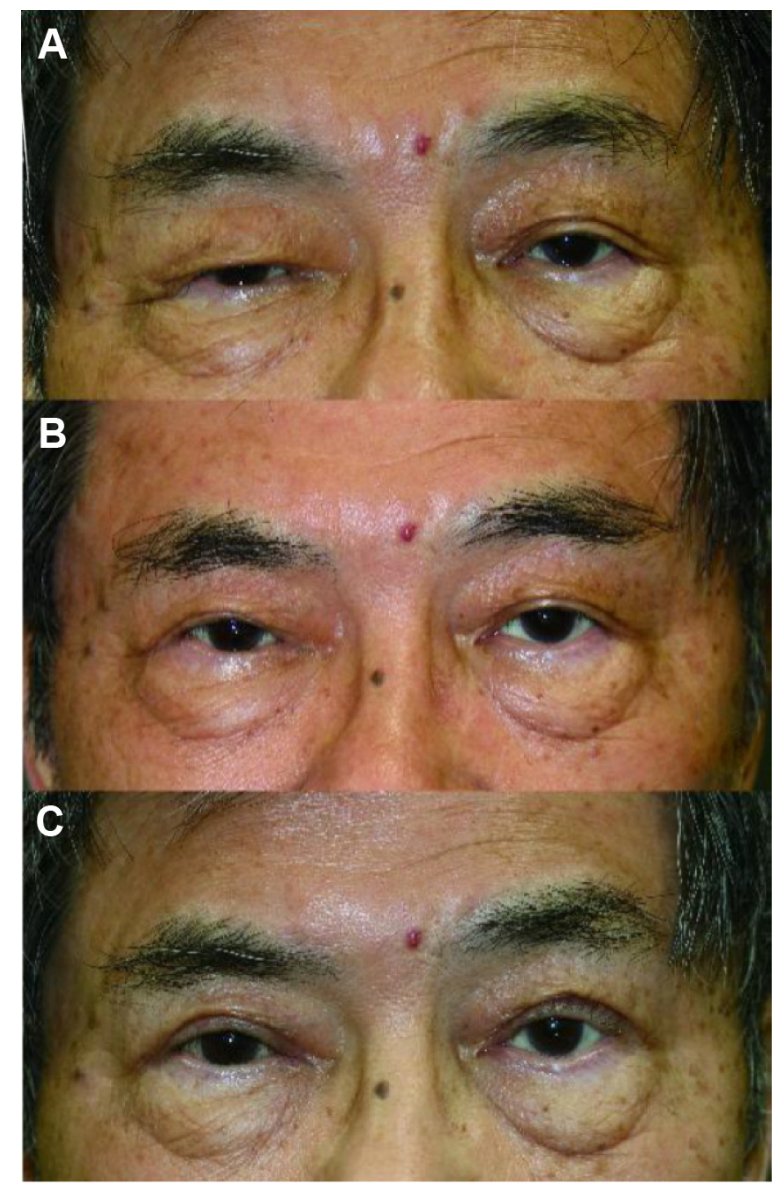

Figure 2 Case 2: A 75-year-old man with right upper blepharoptosis due to myasthenia gravis and brow ptosis due to incomplete facial palsy. (A) Preoperative condition showing the right upper eyelid and eyebrow drooping. (B) One year after external levator advancement. Although concomitant brow elevation surgery had been advocated, the patient declined. (C) Three months after additional bilateral upper eyelid skin excision surgery.

Note: Written consent was obtained from the patient.

concerned about postoperative downtime, external levator advancement with skin resection was performed. The patient's MRD improved to $2.5 \mathrm{~mm} 1$ year postoperatively (Figure 2B). Following satisfactory outcome, the patient desired to achieve the maximum effect of eyelid elevation, including on his normal left side. Hence, additional bilateral upper eyelid skin excision was performed 25 months after the initial surgery, with a satisfactory result noted 3 months postoperatively (Figure 2C).

\section{Case 3}

A 60-year-old man was diagnosed with $\mathrm{MG}$ after developing left blepharoptosis. MGFA grade was 1 (QMG score of 8, at its most severe). After failing to respond to medical treatment including AchE-I, prednisolone, and tacrolimus, persistent blepharoptosis developed. Levator function was 0 $\mathrm{mm}$, and MRD was $-2 \mathrm{~mm}$ (Figure 3A). At 77 years of age,
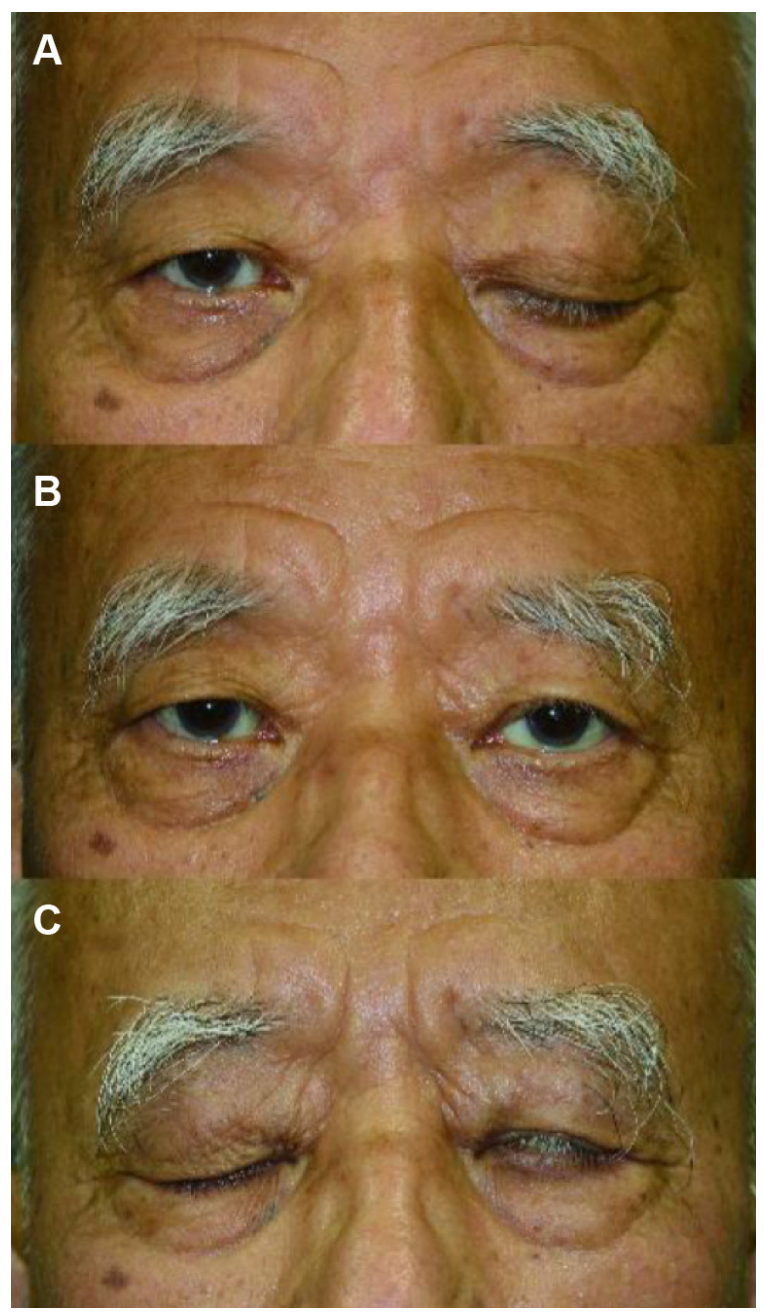

Figure 3 Case 3: A 77-year-old man with left upper blepharoptosis. (A) Preoperative condition. (B) Three years after frontal suspension using autogenous fascia lata. (C) Although mild incomplete eyelid closure is noted, corneal exposure keratopathy did not develop.

Note: Written consent was obtained from the patient.

a frontal suspension procedure using autogenous fascia lata with upper eyelid skin excision was performed. The patient's MRD improved postoperatively to $3.5 \mathrm{~mm}$, and a cosmetically symmetric eyelid shape was achieved in the primary gaze position (Figure 3B). The patient responded as "very satisfied" with the surgical outcome in the questionnaire. Although mild lid lag with incomplete eyelid closure was noted postoperatively, corneal exposure keratitis did not develop (Figure 3C).

\section{Case 5}

A 74-year-old man with moderate diabetes mellitus was diagnosed with $\mathrm{MG}$ after developing right blepharoptosis. MGFA grade was 1 (QMG score of 5, at its most severe). AchE-I therapy improved but did not resolve the blepharoptosis. Prednisolone was not administered owing to 
diabetes mellitus. Levator function was $8 \mathrm{~mm}$, and MRD was $0.5 \mathrm{~mm}$ in the most severe condition (Figure 4A). At 77 years of age, nonincisional transconjunctival levator advancement was performed since the patient preferred a less invasive procedure without skin incision. Care was taken not to elevate the eyelid too high since the patient demonstrated a weak Bell's phenomenon (Figure 4B). Although slight recurrent blepharoptosis was noted 1 year postoperatively, MRD had improved to $2.0 \mathrm{~mm}$ (Figure 4C). The patient did not demand additional surgery and reported satisfaction with the result.

\section{Case 7}

A 75-year-old woman was diagnosed with MG after developing bilateral upper eyelid ptosis. MGFA grade was 3a (QMG score of 17, at its most severe). Although the patient had taken AchE-I, prednisolone, and cyclosporine for more than 2 years, her bilateral blepharoptosis did not improve. Levator function was $9 \mathrm{~mm}$, and MRD was $2.0 \mathrm{~mm}$ bilaterally (Figure 5A). The patient did not demonstrate Bell's phenomenon, although external ocular muscle movement was not impaired.

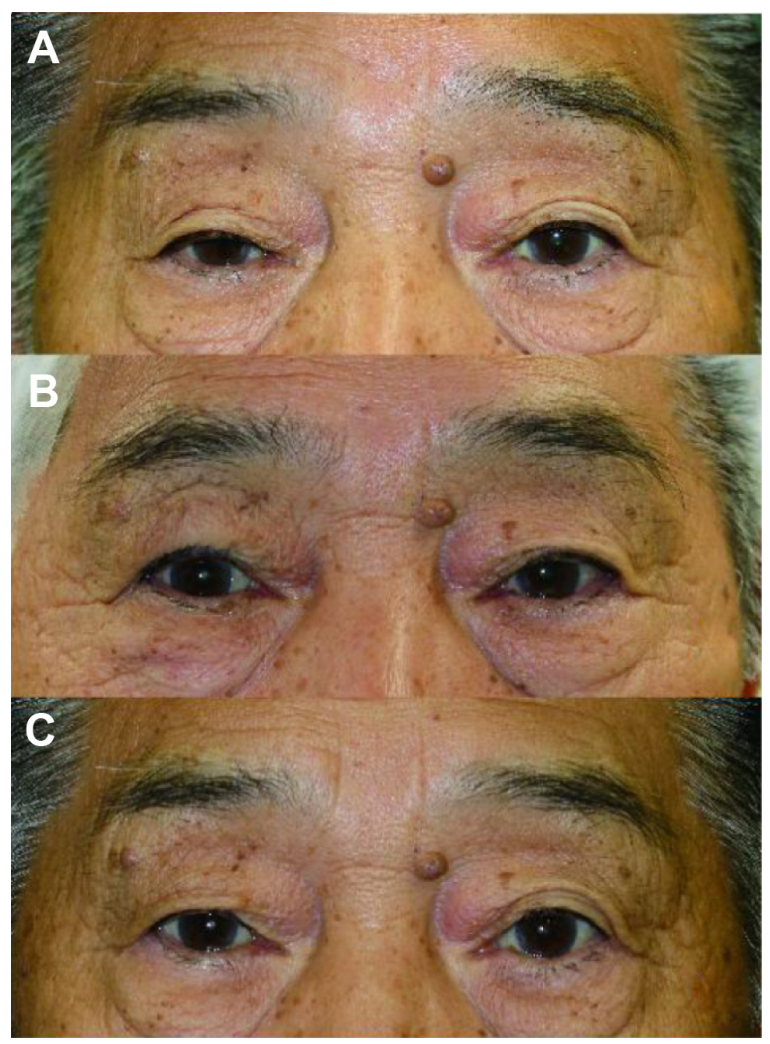

Figure 4 Case 5: A 77-year-old man with right upper blepharoptosis and weak Bell's phenomenon. (A) Preoperative condition. (B) Immediately after the nonincisional levator advancement operation. Eye swelling was minimal. (C) Mild recurrent blepharoptosis was noted I year postoperatively.

Note: Written consent was obtained from the patient.
At 78 years of age, a subbrow skin excision $(13 \times 52 \mathrm{~mm})$ with orbicularis oculi muscle tucking was performed. Although the MRD did not change, and prolonged scar redness persisted nearly 1 year after the operation (Figure $5 \mathrm{~B}$ ), the patient felt "easier" eyelid opening at both short( $\leq 1$ year) and long-term ( $>1$ year) follow up.

\section{Case 8}

A 47-year-old woman was diagnosed with MG after developing bilateral upper eyelid ptosis. MGFA grade was 3a (QMG score of 21, at its most severe). Although several medical treatments were administered, right external ophthalmoplegia with severe blepharoptosis, and mild left blepharoptosis did not improve. At 77 years of age, the patient demonstrated fixed heterophoria (Figure 6A). Furthermore, the right eye was blinded due to optic neuritis (etiology unknown), and Bell's phenomenon was absent. Levator function was $0 \mathrm{~mm}$ in the right eye and $10 \mathrm{~mm}$ in the left, while MRD was 0 and $3.0 \mathrm{~mm}$, respectively. Although the right eye had lost visual function, the patient desired blepharoptosis surgery for esthetic purposes. Hence, frontal suspension, using nylon sutures, with upper eyelid skin resection was performed on the right eye, while external levator advancement was performed on the left eye. In the right eye, care was taken not to excessively elevate the eyelid, to avoid postoperative corneal

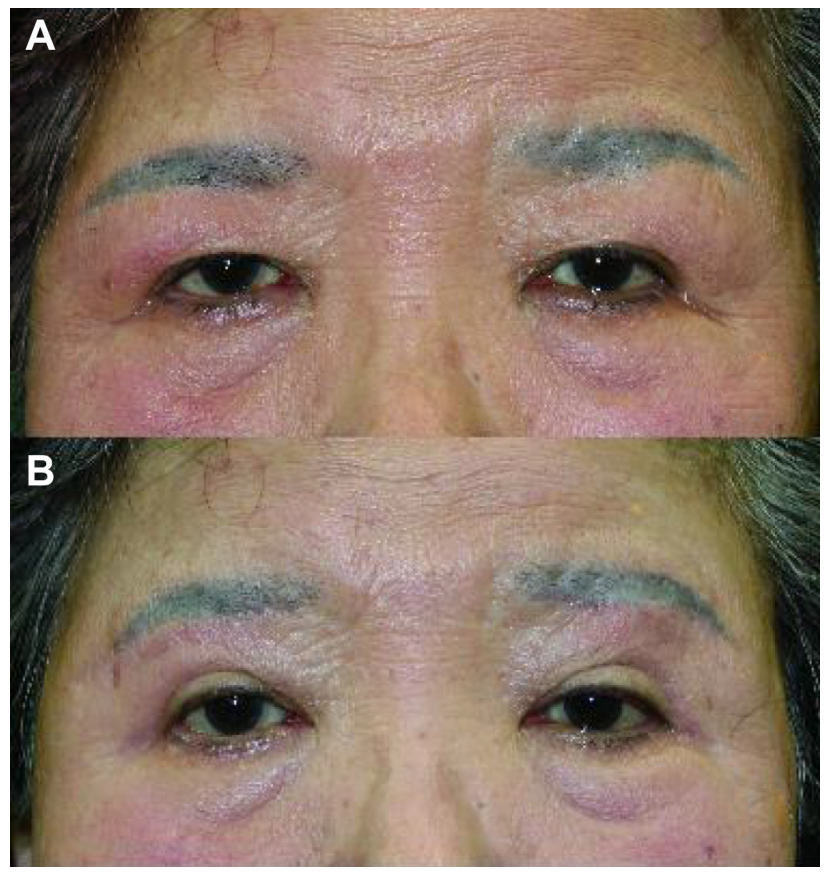

Figure 5 Case 7: A 78-year-old woman with bilateral blepharoptosis and absence of Bell's phenomenon. (A) Preoperative condition. (B) Although prolonged redness of the scar was noted even I year postoperatively, the patient felt easier eyelid opening.

Note: Written consent was obtained from the patient. 


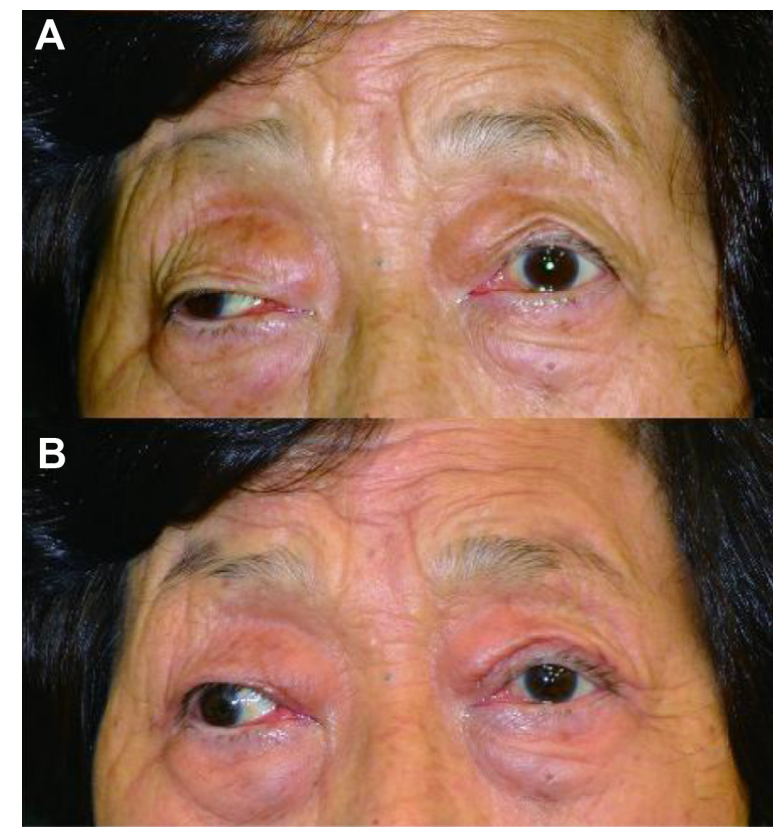

Figure 6 Case 8: A 77-year-old woman with right severe blepharoptosis and heterophoria. (A) Preoperative condition. (B) One year after right frontal suspension using nylon sutures, and left external levator advancement. Although the heterophoria became more apparent, diplopia did not develop since the right eye had been blinded due to persistent optic neuritis.

Note: Written consent was obtained from the patient.

exposure. Although the heterophoria became more apparent (Figure 6B), the patient felt "much easier" eyelid opening in the left eye and was "very satisfied" with both the esthetic and functional outcome. Since the right eye was nonfunctional, diplopia did not develop postoperatively.

\section{Discussion}

Blepharoptosis substantially reduces the quality of life of patients with MG. ${ }^{20-22}$ Despite multiple medical treatments, patients often experience longstanding residual blepharoptosis. In such cases, surgical treatment may play an important role. However, lack of information about the efficacy and adverse effects of blepharoptosis surgery may cause many neurologists to hesitate to inform their longstanding patients about this surgical option.

Generally, blepharoptosis surgery is not very difficult for trained surgeons, and the rate of major functional complications, such as lagophthalmos and exposure keratopathy, is usually not high. Moreover, possible complications can be effectively managed by conservative therapy or additional surgeries if necessary. Bradley et al reviewed the surgical outcome of 16 blepharoptosis procedures in ten patients with MG and concluded that blepharoptosis surgery can be an appropriate treatment option for MG refractory to medical treatment. ${ }^{2}$ However, the indications and specific criteria for blepharoptosis surgery in patients with MG remain unclear and should be established.

The present study presents four surgical criteria according to the specific characteristics of MG. First, the patient's general condition must be considered since clinical manifestations of $\mathrm{MG}$ are variable, ranging from ocular myasthenia to myasthenic crisis, in which patients experience life-threatening respiratory insufficiencies. ${ }^{23}$ Second, medical treatment with strict follow up must be undertaken by an attending neurologist for at least 2 years before surgery. It is reported that $50 \%$ to $60 \%$ of patients who initially present with ocular symptoms progress to develop generalized muscle weakness and that the vast majority will do so within the first 1 to 2 years of diagnosis. ${ }^{6-8}$ Third, blepharoptosis should be consistent with minimal circadian change; otherwise, the surgical effect could collapse. Finally, the patient's quality of life should certainly be weighed, with neurologists and surgeons carefully considering a patient's quality of vision. While the criteria may vary by institute, the development of uniform criteria should be fostered.

Levator function at the most severe condition of MG should be the primary factor in surgical procedure decision making. In addition, MRD, skin condition, eyebrow height, lash-brow distance, orbital shape, coexisting disease, and medication use should also be considered. A patient with poor levator function ( $\leq 4 \mathrm{~mm}$ ) should be treated by frontal suspension. However, the procedure may also be used in cases wherein the levator function is currently $>4 \mathrm{~mm}$, but a decrease in function is expected. Thus, patients with moderate levator function $(5-8 \mathrm{~mm})$ can be treated by either levator advancement or frontal suspension. Alternatively, a patient with at least a $2.0 \mathrm{~mm}$ MRD and thick eyelid skin can be treated by subbrow blepharoplasty with orbicularis oculi muscle tucking. Although subbrow blepharoplasty generally cannot directly improve MRD, it is advantageous in that it is typically associated with short downtime compared with levator advancement and imposes less risk of developing corneal exposure keratopathy. In the case of levator advancement, "external" and "nonincisional" procedures may be an option. Both procedures share the same principle that advancement of the levator aponeurosis and muscle eventually elevates the upper eyelid. However, we generally apply an external procedure for candidates who desire to reduce the upper eyelid skin weight by excision, to achieve the maximum effect of eyelid elevation. In contrast, we employ a nonincisional procedure for candidates who desire a minimally invasive operation with less downtime. 
This study demonstrates the efficacy of blepharoptosis surgery for patients with MG. In terms of functional outcome, MRD was improved 1 year postoperatively in all cases except for case 7 . In case 7, although MRD did not improve, the patient felt easier eyelid opening. No patients developed postoperative exposure keratitis or underwent surgery for overcorrection. Although mild lid lag with incomplete eyelid closure was noted in one patient, it did not become problematic. In terms of esthetic outcome, eyelid scarring was "very minimal", except in patient 7 who had "minimal" scarring after subbrow skin excision. Additionally, the scar in this case demonstrated redness for a relatively longer period, taking more than 1 year to subside. This patient's poor wound healing ability was likely due to daily use of prednisolone and cyclosporine. Five patients had "no" eyelid asymmetry and one had "subtle" asymmetry. Although two patients (MGFA 2a and 3a) demonstrated "obvious" asymmetries, these were evident before surgery and were not caused by the surgery. In terms of overall satisfaction, seven patients were "very satisfied" and one patient was "satisfied" with the result. No patients were "unsatisfied."

It should be noted that particular precautions must be taken during these operations, in order to avoid postoperative lagophthalmos or corneal exposure keratitis. This is important since such patients often show the absence of Bell's phenomenon, owing to external ophthalmoplegia. In patients with heterophoria, the risk of postoperative worsening of diplopia must also be considered. In such cases, eyelid elevation should be corrected to an appropriate extent and not overcorrected.

The major limitation of the present study is the relatively small number of patients who actually underwent the operation. We are considering future studies with a greater number of patients and with longer follow-up periods.

In conclusion, blepharoptosis surgery for patients with MG may be a good choice of treatment if performed with appropriate surgical criteria and procedure decision making. The surgery is especially effective for patients who have residual blepharoptosis despite multiple medical treatments. Therefore, in order to increase the quality of life of patients with $\mathrm{MG}$, we recommend that neurologists and surgeons collaborate more systematically and discuss comprehensive treatment plans.

\section{Acknowledgments}

This work was supported by a grant from the Japanese Ministry of Education, Science, Sports, and Culture (grant number 23591255) and a Neuroimmunological Disease Research Committee grant from the Japanese Ministry of Health, Labour, and Welfare.

\section{Disclosure}

The authors report no conflicts of interest in this work.

\section{References}

1. Kapetansky DI. Surgical correction of blepharoptosis in myasthenia gravis. Am J Ophthalmol. 1972;74(5):818-820.

2. Bradley EA, Bartley GB, Chapman KL, Waller RR. Surgical correction of blepharoptosis in patients with myasthenia gravis. Ophthal Plast Reconstr Surg. 2001;17(2):103-110.

3. Carter SR, Meecham WJ, Seiff SR. Silicone frontalis slings for the correction of blepharoptosis: indications and efficacy. Ophthalmology. 1996;103(4):623-630.

4. Yu CC, Chen SG, Chen TM. Frontalis slings with palmaris tendon as an adjuvant treatment for myasthenic blepharoptosis: a case report. Ann Plast Surg. 2007;58(5):577-579.

5. Asamura S, Kakizaki H, Enjyo M, Hashimoto T, Isogai N. Frontalis sling procedure for ocular myasthenia gravis. Clin Ophthalmol. 2012; 6:575-577.

6. Benatar M, Kaminski H. Medical and surgical treatment for ocular myasthenia. Cochrane Database Syst Rev. 2012;12:CD005081.

7. Bever CT Jr, Aquino AV, Penn AS, Lovelace RE, Rowland LP. Prognosis of ocular myasthenia. Ann Neurol. 1983;14(5):516-519.

8. Oosterhuis HJGH. Myasthenia Gravis. Groningen: Groningen Neurologic Press; 1997.

9. Benatar M, Kaminski HJ; Quality Standards Subcommittee of the American Academy of Neurology. Evidence report: the medical treatment of ocular myasthenia (an evidence-based review): report of the Quality Standards Subcommittee of the American Academy of Neurology. Neurology. 2007;68(24):2144-2149.

10. Osserman KE. Ocular myasthenia gravis. Invest Ophthalmol. 1967; 6(3):277-287.

11. Sghirlanzoni A, Peluchetti D, Mantegazza R, Fiacchino F, Cornelio F. Myasthenia gravis: prolonged treatment with steroids. Neurology. 1984;34(2):170-174.

12. Evoli A, Batocchi AP, Minisci C, Di Schino C, Tonali P. Therapeutic options in ocular myasthenia gravis. Neuromuscul Disord. 2011;11(2):208-216.

13. Kaminski HJ, Daroff RB. Treatment of ocular myasthenia: steroids only when compelled. Arch Neurol. 2000;57(5):752-753.

14. Roach ES. Treating ocular Myasthenia Gravis with inadequate evidence. Arch Neurol. 2007;64(12):1794-1795.

15. Nagane $Y$, Utsugisawa $K$, Suzuki S, et al. Topical naphazoline in the treatment of myasthenic blepharoptosis. Muscle Nerve. 2011;44(1):41-44.

16. Shimizu Y, Suzuki S, Utsugisawa K, et al. Is surgical intervention safe and effective in the treatment of myasthenic blepharoptosis? A multicenter survey in Japan. Eur Neurol. 2014;71(5-6):259-261.

17. Carraway JH, Vincent MP. Levator advancement technique for eyelid ptosis. Plast Reconstr Surg. 1986;77(3):394-403.

18. Shimizu Y, Nagasao T, Asou T. A new non-incisional correction method for blepharoptosis. J Plast Reconstr Aesthet Surg. 2010;63(12): 2004-2012.

19. Jaretzki A, Barohn RJ, Ernstoff RM, et al. Myasthenia gravis: recommendations for clinical research standards. Task Force of the Medical Scientific Advisory Board of the Myasthenia Gravis Foundation of America. Neurology. 2000;55(1):16-23.

20. Paul RH, Nash JM, Cohen RA, Gilchrist JM, Goldstein JM. Quality of life and well-being of patients with myasthenia gravis. Muscle Nerve. 2011;24(4):512-516.

21. Mullins LL, Carpentier MY, Paul RH, Sanders DB; Muscle Study Group. Disease-specific measure of quality of life for myasthenia gravis. Muscle Nerve. 2008;38(2):947-956.

22. Masuda M, Utsugisawa K, Suzuki S, et al. The MG-QOL15 Japanese version: validation and associations with clinical factors. Muscle Nerve. 2012;46(2):166-173.

23. Lacomis D, Silvestri NJ, Wolfe GI. What's in the Literature? J Clin Neuromuscul Dis. 2014;16(1):32-41. 
Clinical Ophthalmology

\section{Publish your work in this journal}

Clinical Ophthalmology is an international, peer-reviewed journal covering all subspecialties within ophthalmology. Key topics include: Optometry; Visual science; Pharmacology and drug therapy in eye diseases; Basic Sciences; Primary and Secondary eye care; Patien Safety and Quality of Care Improvements. This journal is indexed on

PubMed Central and CAS, and is the official journal of The Society of Clinical Ophthalmology (SCO). The manuscript management system is completely online and includes a very quick and fair peer-review system, which is all easy to use. Visit http://www.dovepress.com/ testimonials.php to read real quotes from published authors. 